Voix et Images

\title{
Anamorphose, perception carnavalisante et modalités polyphoniques dans Trou de mémoire
}

\section{M.-Pierrette Malcuzynski}

Volume 11, numéro 3 (33), printemps 1986

Yolande Villemaire

URI : https://id.erudit.org/iderudit/200582ar

DOI : https://doi.org/10.7202/200582ar

Aller au sommaire du numéro

Éditeur(s)

Université du Québec à Montréal

ISSN

0318-9201 (imprimé)

1705-933X (numérique)

Découvrir la revue

Citer cet article

Malcuzynski, M.-P. (1986). Anamorphose, perception carnavalisante et modalités polyphoniques dans Trou de mémoire. Voix et Images, 11(3), 475-494. https://doi.org/10.7202/200582ar d'utilisation que vous pouvez consulter en ligne.

https://apropos.erudit.org/fr/usagers/politique-dutilisation/ 


\title{
Anamorphose, perception carnavalisante et modalités polyphoniques dans Trou de mémoire
}

\author{
par M.-Pierrette Malcuzynski, Université de Varsovie \\ À André Belleau, instigateur principal et perspicace d'une \\ approche bakhtinienne aux textes littéraires québécois.
}

\section{Avant-propos}

Cet article reprend quelques-uns des problèmes de théorie narrative discutés à l'origine dans une thèse de $\mathrm{Ph}$. D. en littérature comparée (soutenue à l'Université $M^{\circ}$ Gill en 1981), et dont certains d'entre eux ont été retravaillés dans une série d'articles' ${ }^{1}$. Il s'agit surtout ici d'une analyse socio-textuelle de Trou de mémoire et qui, dans une optique sociocritique se démarquant des prémisses qui relèvent d'une sociologie de la littérature plus traditionnelle, entend tenir compte de l'objectif principal posé par Claude Duchet: celui de travailler le statut du social dans le texte plutôt que définir le statut social du texte. C'est bien la problématique que nous suggère globablement les théories de Mikhaïl Bakhtine sur le roman et que je chercherai ici à prolonger et à spécifier en termes critiques.

Les romans d'Hubert Aquin ont été et continuent d'être l'objet de très nombreuses études touchant à une multitude de problématiques comme en témoigne l'important recensement bibliographique en cours ${ }^{2}$. D'autre part, il ne s'agit pas de revenir sur les longues définitions du carnavalesque et du polyphonique, mais plutôt de cerner quelques-unes de leurs conséquences romanesques contemporaines, à partir de Trou de mémoire et du phénomène de l'anamorphosisation en tant que pratique esthétique. Ce que je propose est plus exactement une discussion portant sur les implications théoriques et méthodologiques que cette pratique entraîne pour la critique littéraire. Après avoir décrit et précisé l'apport de l'anamorphose à l'esthétique du roman, ainsi que sa technique pour ce qui est de la mise en texte, je chercherai à en cerner les tracés idéologiques, et les formations socio-historiques auxquelles cette pratique renvoie. Ainsi, il ne s'agit pas de produire une "interprétation " de Trou de mémoire mais, à l'instar de la pensée de Bakhtine, de pratiquer une recherche d'application en termes critiques du'concept d'esthétique dans la littérature par des définitions qui tiennent compte des autres domaines, dans l'unité de la production culturelle. L'enjeu est d'étudier Trou de mémoire sur des bases à la fois englobantes et spécifiques, en précisant le concept même d'esthétique littéraire dans l'optique de la coexistence et de l'interaction de pratiques culturelles différentes. 
Enfin, cet article se voudrait une modeste réponse à l'aimable invitation d'André Belleau dans son article «Carnavalisation et roman québécois» ${ }^{3}$.

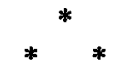

Anamorphose. L'origine étymologique du mot - du grec ANA, de bas en haut ou en arrière, et MORPHE, forme - signale une forme que l'on perçoit dans l'angle optique qui monte du bas vers le haut. Le préfixe marque aussi l'achèvement ou la décomposition. Anamorphose: forme décomposée jusqu'au moment du redressement de perspective, recomposition de la forme "en la remontant» du regard. Au facteur de la représentation de la forme s'ajoute celui de sa "lecture»: le spectateur doit jouer un rôle et re-former l'image lui-même. Cela n'a rien de très nouveau en art: songeons aux peintures illusionnistes des murs et des plafonds, ou encore à l'application de ce principe par l'école hollandaise, appelé perspektyfkas. La participation active du spectateur à la reconstruction de l'image est ce qu'il importe de remarquer ici. Rappelons également que le mot "anamorphose" est introduit dans la seconde moitié du XVII ${ }^{\mathrm{e}}$ siècle par un jésuite allemand, Gaspar Schott, longtemps après que la pratique elle-même eut existé dans le domaine de l'optique.

Pour ce qui est de Trou de mémoire ${ }^{4}$, nul ne saurait contester l'influence qu'Hubert Aquin aura subi à la lecture du livre de Jurgis Baltrusaitis, Anamorphoses ou magie artificielle des effets merveilleux, dont la première édition date de 1955. L'auteur y décrit les techniques de l'anamorphose dans le domaine de l'art. Il s'agit d'une

projection de formes hors d'elles-mêmes et leur dislocation de manière qu'elles se redressent lorsqu'elles sont vues d'un point de vue déterminé. Le procédé est établi comme une curiosité technique, mais il contient une poétique de l'abstraction, un mécanisme puissant de l'illusion optique, et une philosophie de la réalité factice. C'est un rébus, un monstre, un prodige. Tout en appartenant au monde des singularités qui, dans le fond humain, a toujours eu un "cabinet", et un refuge, il en déborde souvent le cadre hermétique... Un instrument nouveau, le miroir, fait son apparition dans le domaine anamorphotique vers 1615-25. Géométriquement, c'est la substitution à l'angle visuel de l'angle de réflexion. L'objet lui-même, étincelant, revêt un caractère magique en devenant évocateur de spectres.

Il est tout à fait étonnant de constater à quel point on retrouve le parallèle de ces coordonnées dans Trou de mémoire. En effet, le roman est une projection hors de [lui-même] et [sa] dislocation. Ceci est évident lorsqu'on se rend compte, par exemple, que le "Journal d'Olympe Ghezzo-Quénum» est une réflexion asymétrique du manuscrit autobiographique de Pierre X. Magnant dont les formes, apparemment disloquées, retrouvent une certaine proportion quand elles sont abordées à la lumière d'une perspective déterminée. Le procédé du mécanisme d'illusion optique, le trompe-l'œil, comme dans l'art, 
où il détermine l'essence cachée du tableau de Holbein le Jeune (les Ambassadeurs), désigne une ou des vérités dans l'écriture, dont la véritable signification est masquée derrière une réalité factice. Pour Aquin comme pour ses narrateurs, l'acte d'écrire représente à la fois un refuge et une tentative de libération. Magnant écrit un roman qui est à sa propre image mais qui en déborde et se disloque à travers le journal de Ghezzo-Quénum, de la même manière que l'anamorphose déborde de son cadre hermétique; d'où la tentative de libération et, dans une certaine mesure, la réalisation libératoire. Magnant écrit au début de ses textes: le roman d'ailleurs c'est moi: je me trouble, je me décris, je me vois, je vais me raconter sous toutes les coutures, car il faut bien l'avouer, j'ai tendance à déborder comme un calice trop plein (TdeM 19). Le roman est aussi un refuge parce qu'écrire impose le silence au narrateur, l'empêche de parler; parler pourrait signifier sa perte (TdeM 21). En outre, l'éditeur situe le texte de Magnant hors littérature, hors fiction et tout à fait hors roman ( TdeM 74), ce qui fait éclater les normes du cadre initial du manuscrit romanesque tel que conçu par son narrateur. D'autre part, le miroir du XVII ${ }^{\mathrm{e}}$ siècle confère à l'objet, dans le domaine de l'anamorphose, un caractère magique en devenant évocateur de spectre; spectral, Ghezzo-Quénum l'est par rapport à Magnant. De plus, il avertit qu'il écrit sur une table surmontée d'un miroir qui me renvoie mes mots à l'envers (TdeM 175). De même, Trou de mémoire se constitue comme une série d'évocations miroitantes et artificielles au sens où chaque fait, chaque concept, chaque situation, est en état de mouvement incessant et se trouve perpétuellement au seuil de sa propre annulation, frisant ainsi un phénomène d'implosion.

Esthétiquement, l'anamorphose a pour résultat un processus en deux temps $^{6}$. Le premier relève directement du domaine de l'optique. C'est une illusion créée par un miroir déformant, qui ne renvoie pas une image isomorphe, mais au moyen duquel l'objet se disloque, se disproportionne, ce qui entraine une confusion chez le spectateur. Le deuxième temps a une signification presque transcendante, ressortissant au domaine de la démystification, et constitue un procédé qui touche au processus carnavalesque de «mourir pour renaître" sans toutefois s'y identifier (au sens spécifique de détruire l'illusion pour obtenir la réalité). Cette signification surgit seulement au moment où le point de vue correspondant au redressement de perspective de formes de l'image a été retrouvé, révélant alors celle-ci dans sa totalité et en bonnes proportions. Baltrusaitis souligne l'importance de mettre en valeur, du point de vue esthétique, le côté caché et mystérieux de l'anamorphose: il faut inclure des formes dans d'autres formes. Il faut les déguiser?. Voyons ci-dessous l'esprit dans lequel s'emploie l'anamorphose en dessin ou en peinture (cf. Pratica della perspettiva, 1559) ${ }^{8}$ :

Pour mieux dissimuler ce que l'on peint selon les pratiques indiquées, la peinture qui a à figurer les deux têtes ou autres représentations doit savoir ombrer et recouvrir l'image afin qu'elle montre, au lieu des deux têtes, des pays, des eaux, des murs, des rochers et autres choses diverses... Les figures peuvent être décomposées avec des parties séparées les unes des autres de la façon qu'elles 
paraissent se rejoindre lorsqu'elles sont regardées de biais: aussi le front d'une figure peut être placé en un lieu, le nez en un autre et le menton ailleurs encore... L'on ne reconnaît plus si la peinture représente une tête, mais le nez paraîtrait une chose et le front une autre, et, en particulier, si le peintre sait faire paraitre le nez comme un rocher et le front comme une motte de terre selon qu'il lui semblera.

En ce qui concerne les Ambassadeurs, sa double signification immédiate est conférée par cette qualité de trompe-l'œil: perspective «naturelle» et anamorphose. Occultation et divulgation au sens propre de ces termes; c'està-dire masquer par la lumière, camouflage de sa "vérité», de sa réalité et rendre public ce qui était ignoré de la plupart mais connu de quelques-uns. En art, lorsque appliqué par exemple à des objets religieux, ce qui importe est la révélation miraculeuse de quelque chose de caché. Ou encore, il s'agit de cette nécessité de camoufler des thèmes érotiques ou obscènes dont la représentation des images n'est visible que dans une perspective optique oblique. C'est dans ce même esprit de camouflage (occultation) que RR interprète dans Trou de mémoire le tableau des Ambassadeurs, en lui donnant la configuration spectrale que le crâne anamorphotique appelle:

les représentants d'une puissance étrangère ne représentent rien d'autre que la mort, sol funèbre en forme de crâne, véritable patrie qui n'est pas reconnue comme telle par les chancelleries, mais dont le statut renverse à jamais les canons de la diplomatie mondaine. La mort frappe. Son spectre, indéchiffrable au premier regard, agit avec d'autant plus de force pour multiplier la terreur: la mort, figurée anamorphiquement par Holbein, est toujours subite. Son visage, rallongé ou raccourci par les artifices de la "perspective curieuse», a la sombre beauté d'un masque de mort: quand on le reconnaît, on est aveuglé par ce choc noir qui crève les yeux! Ce qui avait les propriétés de l'immuable et de la présence réelle se trouve réduit en poussière soudaine!

$\left(\right.$ TdeM 133) ${ }^{9}$

Cette interprétation est contestée par l'éditeur dont le récit «Suite et fin» suit immédiatement le texte de RR et précède le "Journal de Ghezzo-Quénum", constituant de la sorte une espèce de surdétermination anamorphotique. C'est dans l'esprit de divulgation que l'éditeur confère au texte de RR le sens de piège optique, sens qui serait propre à la peinture de Holbein.

$A u$ fond, ce tableau de la mort n'est qu'un double terrible de cette réalité fastueuse, luxuriante, éblouissante qui trompe celui qui la regarde de trop près. Ces "Ambassadeurs", ne sont qu'apparences trompeuses, masques gracieux de la mort; et, en cela, ils sont à l'image même d'une vie terrestre dont la laideur n'a d'égale que la séduction de son déguisement... Et j'en viens à me demander si la clé de ce texte ne réside pas dans ce tableau à énigme dont il est si abondamment question dans le dernier chapitre et si, en quelque 
sorte, le texte signé " $R R$ " n'a pas été écrit en trompe-l'ail et de telle sorte que le lecteur non prévenu, ou trop pressé, n'en décèle pas le sens caché sous un fatras d'allégories et de fausses pistes.

(TdeM 138)

L'éditeur interprète les deux figures du tableau de Holbein comme étant Magnant et Joan (TdeM 142). En fait, il s'agit d'un autre trompe-l'œil, car il faudrait plutot lire Magnant et Ghezzo-Quénum. De la même manière que Rachel Ruskin devient l'objet anamorphotique dans le Journal de ce dernier, et RR dans Trou de mémoire, Joan le devient dans les textes de Magnant: blason mortuaire au centre du livre, écrit l'éditeur, Joan fait fonction de crâne indiscernable qui se tient entre les 'ambassadeurs'. Elle anime tout; elle est le foyer invérifiable d'un récit qui ne fait que se désintégrer autour de sa dépouille (TdeM 143).

Remarquons cependant qu'il existe aussi une lecture frontale de l'énigme des Ambassadeurs ( si énigme il y a vraiment), rendue possible si l'on tient un tube d'une certaine dimension à bout de bras, face au tableau. Si l'on oriente le tube obliquement entre la cavité nasale et l'orbite gauche du crâne anamorphotique, explique l'historien de l'art Jean-Louis Ferrier, alors on voit apparaître, à l'intérieur de celui-ci, un second crâne, plus petit, enroulé dans le premier. C'est un deuxième détournement de sens... Holbein a caché une image à l'intérieur de son image cachée ${ }^{10}$. Il est important de souligner ce détail car c'est ce qu'effectue Hubert Aquin, on le verra. Comme le remarque un autre historien de l'art, John Berger, the eye moves from fur to silk to metal to wood to velvet to marble to paper to felt... The two men have a certain presence and there are many objects which symbolize ideas, but it is the materials, the stuff, by which the men are surrounded and clothed which dominate the painting"l. Pour ce qui est du crâne anamorphotique, Berger indique que les diverses théories à son sujet sont toutes d'accord au moins sur un point, à savoir qu'il s'agit d'un momento mori: un jeu sur le motif médiéval du crâne comme rappel constant de la présence de la mort. Mais il ajoute ce fait significatif, c'est qu'il ait été peint justement dans une optique différente de tout le reste. Ceci renvoie en particulier au violent contraste qu'il constitue par rapport aux détails mimétiquement minutieux des autres objets représentés dans le tableau. Pris sous cet angle, l'anamorphose est esthétiquement symptomatique du style artistique de l'époque à laquelle Holbein peint son chef-d'œuvre, le maniérisme. Sans aller dans les détails, il convient toutefois de noter que le programme théorique que propose le maniérisme n'est pas seulement axé sur les moyens par lesquels l'artiste choisit les méthodes les plus aptes à subvenir à ses besoins artistiques, mais qu'il repose également sur des objectifs précis. Le maniérisme est le premier style artistique à se préoccuper du problème culturel qui consiste à concevoir la relation entre tradition et innovation - soit la notion de changement, de développement comme un problème devant être résolu par des moyens rationnels ${ }^{12}$. Ceci peut paraître paradoxal en ce qui concerne l'effet esthétique produit par l'anamorphose, mais c'est justement au niveau de la pratique que l'anamorphosisation justifie dans le domaine de l'art plastique les contradictions et les 
tensions socio-historiques que, au niveau du style, le maniérisme vient marquer à l'égard de son époque. Or, rappelons brièvement qu'il s'agit d'une période de l'histoire où l'Europe occidentale est en pleine transformation; c'est l'époque de la réorientation des routes commerciales vers l'Atlantique et la mise en place systématique de l'empire européen colonisateur dans les Amériques. Une période marquée par de profonds bouleversements sociaux et culturels (religieux, scientifiques, intellectuels) engendrés par la crise économique qui sévit dans le bassin méditerranéen et se répercute à travers le reste de l'Europe.

Ce serait, par contre, une absurdité historique que de penser ici à un paradigme épistémologique entre les conditions socio-historiques de la deuxième moitié $d u X V I^{c}$ siècle européen et celles du Québec de la deuxième moitié du XXe, et dont le dénominateur commun s'expliquerait alors dans le domaine artistique par une sorte de possibilité esthétique, l'anamorphose. Du reste, le personnage de l'éditeur dans Trou de mémoire nòus met en garde indirectement contre ce genre de substitution comparative en rendant manifeste le type de réflexion réductrice qui en résulterait: le piège optique, dans les 'Ambassadeurs', ne fait que reproduire dans le domaine artistique le piège inhérent à la création elle-même (TdeM 138). Et si, toujours pour l'éditeur, les Ambassadeurs est un tableau en forme d'histoire de meurtre [...], en quelque sorte, le double antérieur du roman (TdeM 144), ceci renvoie à la structure diégétique de Trou de mémoire. En effet, le processus de dédoublement y a pour fonction importante de mettre en rapport ce que RR constitue pour l'ensemble de Trou de mémoire avec l'effet que produit le crâne anamorphotique dans les Ambassadeurs. Il n'est pas afortuiti que ce soit RR qui introduise dans le roman le tableau de Holbein. Sous cet angle, les Ambassadeurs fonctionne comme une sorte de double antérieur à son récit, tout comme l'est celui-ci par rapport au récit de Magnant:

$R R$ ne sont pas vraiment mes initiales; c'est en quelque sorte un pseudonyme abrégé dont je me suis affublée et qui n'est pas sans exprimer ma volonté initiale de me situer d'emblée au niveau de la fiction. Oui, je n'ai pas cessé de poursuivre - dans cet écrit polymorphe - une expérience d'écriture fictive...

(TdeM 123)

Cela me paraît en revanche une erreur méthodologique de chercher à identifier la structure narrative de Trou de mémoire dans les mêmes termes, c'est-à-dire comme une série de structures qui se dédoublent les unes les autres. Ceci reviendrait au même que chercher le "double antérieur" de l'histoire du Québec contemporain dans la société européenne des $\mathrm{XVI}^{\mathrm{e}}$ et $\mathrm{XVII}^{\mathrm{e}}$ siècles et en arriver ainsi à expliquer, par exemple, la forme et le contenu «baroques» du roman. N'oublions pas que le trompe-l'œil fait partie intégrante de Trou de mémoire; il en est l'une des composantes textuelles constitutives dont la caractéristique fondamentale est ici, à mon sens, le redoublement. Il s'agit d'un phénomène qui s'oppose à celui du "double" deux entités de formes exactement identiques mais de natures distinctes - et à celui du «dédoublement", qui renvoie soit à la duplication d'une seule 
entité, soit à un même sujet qui a deux personnalités différentes (respectivement, Magnant versus $R R$ et $R$ [achel] $R$ [ uskin], mais justement les deux redoublés d'un trompe-l'œil). Le redoublement peut être défini en un premier temps, esthétiquement, comme la réflexion déformée d'une entité perçue dans un miroir convexe (telle que l'anamorphose). Par rapport à son modèle, le «redouble» n'est pas son même non-identique (ce qui rejoindrait le dédoublement de la personnalité au sens psychanalytique). Il s'agit plus exactement d'une entité qui a sa propre identité; c'est-à-dire qui possède des caractéristiques et des propriétés particulières par rapport à celles d'une autre identité dont certains détails déformés lui sont néanmoins similaires. Le deuxième sens de redoublement, également applicable à Trou de mémoire presque sous forme métaphorique, est celui de remettre en doublure, comme dans «doubler une robe". Les vêtements somptueux des Ambassadeurs sont, ici, phrases opaques dont on habille la nudité putrescente de Joan, écrit l'éditeur au sujet du texte de RR qu'il compare ainsi à la texture des Ambassadeurs:

Le drapé des hyperboles et des paradigmes remplace les tissus damassés et les soies chinées du tableau de Holbein le Jeune. L'agencement même du livre - cette séquence qui va de la confession à la création romanesque - peut se comparer à la composition de Holbein: c'est un blason écrit sur fond de mort mais en trompe-l'ail.

(TdeM 143)

Il y a aussi un troisième sens à redoublement au sujet duquel je m'attarderai ultérieurement. Celui qui renvoie à accroissement, à augmentation et, par extension, à accumulation et surenchère. Je voudrais par contre m'arrêter un instant sur quelques exemples de la première définition.

Trou de mémoire repose sur tout un système diégétique en forme d'oppositions et de contradictions thématiques dont la constante mise en rapport les unes aux autres a pour effet leurs déformations. La nature dialogique de cette mise en rapport est évidente dès le début du roman. La première partie du livre constituée par la lettre qu'Olympe Ghezzo-Quénum adresse de la Côte d'Ivoire à son lointain collègue québécois, Pierre X. Magnant, est bel et bien une invitation au dialogue. Il lui écrit qu'il est aussi pharmacien et s'efforcera d'employer des notions externes à la relation de fraternité qui, pour [lui] du moins, s'est établie entre $[$ eux $]$ (TdeM 9). Ghezzo-Quénum se proclame incroyablement frère de Magnant mais non son jumeau (ni son double); Magnant est un artisan de la révolution dans son pays alors que Ghezzo-Quénum ne travaille qu'humblement à la révolution du sien. Et si Bakounine et Thomas de Quincey sont les deux seuls écrivains blancs que [Ghezzo-Quénum] vénère, ils sont, légèrement différemment, les auteurs préférés de Magnant (TdeM 9). L'un prononce un discours, un hymne révolutionnaire, lors d'un Congrès politique tenu à Montréal le 24 juillet 1966, alors que l'autre l'adresse lors d'un meeting révolutionnaire à Abidjan le 28 juillet de cette même année.

Voici donc quelques exemples de correspondances imparfaites, à partir desquelles le lecteur peut alors lui-même continuer d'effectuer des rappro- 
chements avec d'autres éléments du roman. Ainsi, Rachel Ruskin, peut être RR et en tant que telle, maîtresse de Ghezzo-Quénum - j'ai fait la connaissance d'une infirmière du Lagos General Hospital; plus précisément, elle est directrice-adjointe de la pharmacie. Elle est originaire de Montréal et m'a assuré vous connaître, écrit Ghezzo-Quénum dans sa lettre (TdeM 14). Elle est enceinte de Magnant, écrivain à ses heures, et éditrice de la totalité du livre sous le pseudonyme RR. Elle est aussi la sour de Joan, qui, elle, est microbiologiste à Montréal et la maîtresse de Magnant, mais également spécialiste en esthétique de théâtre en tant que «Joan» et amante de RR. Joan - symbole du cadavre du Québec - est droguée par Magnant avant d'être assassinée par lui; RR qui, au contraire, symbolise la renaissance du pays, est droguée par Ghezzo-Quénum après avoir été violée. Abidjan est le redoublement géographique de Montréal; la ville de Lagos et le Golf de Guinée le sont par rapport à Ouchy, le port de la ville de Lausanne sur le Lac Léman en Suisse Romande; Ouchy (j'allais écrire: Lagos!), s'exclame Ghezzo-Quénum (TdeM 15), et plus tard, on ne voit qu'un écran de pluie opaque au lieu des Alpes: le lac s'est transformé en mer noire (TdeM 15), allusion au Golf de Guinée, centre africain du trafic négrier aux XVI ${ }^{\mathrm{e}}$ et XVII ${ }^{e}$ siècles, surnommé de la sorte la Côte des Esclaves. Et ainsi de suite.

Ce ne sont que quelques illustrations des procédés asymétriques de redoublements qui se répercutent aussi au niveau de la structure narrative du roman. Mise à part une exception - Joan, qui n'a pas le droit de parole à part entière --, tous les personnages font revivre par leurs textes respectifs certains faits présentés par ceux de leurs "correspondants», mais de manière imparfaite, en les modifiant. Il s'agit d'une série de polarisations narratives, lesquelles, au contact les unes aux autres, remettent chaque fois en question la validité des discours auxquels réfère l'ensemble de l'œuvre singulière, non seulement au niveau significatif mais également à celui de la forme, de la mise en situation et des actions ainsi que de la caractérisation des personnages.

Plus exactement, on devrait parler en termes de modalités polyphoniques dans Trou de mémoire, constituées de plusieurs instances narratives, en apparence autonomes, mais qui finissent par s'auto-détruire par leurs contradictions: le principe de désaffirmation, ou mieux dit, le sympôme de l'impossibilité de s'affirmer qui se manifeste à tous les niveaux de la textualisation. Chaque énoncé, chaque sujet énonciateur particulier est certes autonome en son individualité mais celle-ci n'acquiert ses particularités que par la mise en relation de son propre discours avec ceux des autres sujets. D'importance égale dans la diégèse, équipollent, dirait Bakhtine, chacun des je-narratifs croit ainsi tenir la clé significative du texte qu'il précède ou qu'il commente. Pour l'éditeur, la clé est dans le texte de RR à travers le tableau des Ambassadeurs; dans "L'incident de Neptune", il complète même les manuscrits de Magnant en y ajoutant du sien, ce qui modifie en quelque sorte l'ensemble des récits. Ghezzo-Quénum croit, pour sa part, découvrir la véritable identité de Charles-Édouard Mullahy:

je sais que $R R$ est enceinte de vous. Je sais aussi que vous avez assassiné Joan et que vous vous définissez volontiers comme révo- 
lutionnaire... C'est sans doute à cause de votre activité subversive que vous vous êtes transformé en éditeur nommé Mullahy. Et toute cette histoire autour de la publication de l'autobiographie de P.X. Magnant n'est qu'une manouvre de diversion et une feinte pour permettre à votre "ami», une fois mort et enterré, de ressusciter pour ceuvrer plus sûrement dans la parfaite clandestinité de la mort...

(TdeM 200)

Mullahy ne serait ainsi qu'un autre redoublement de Ghezzo-Quénum - Magnant, doublement redoublé en se cachant derrière la signature de l'éditeur / RR (je renvoie au détail du petit crâne à l'intérieur du crâne anamorphotique des Ambassadeurs). Par ailleurs, il est fait ici une allusion directe à Magnant lorsqu'il est question de son Cahier noir: Ghezzo-Quénum s'exclame, je vois tout en noir (TdeM 200). Et enfin, RR, sigle multiple dissimulant en ses propres initiales le nom et l'identité de Rachel Ruskin et qui, après s'être emparée du moi-individu ainsi que du je-narratif de Magnant, semble vouloir récupérer tous les discours des autres à la fin du roman, sans toutefois les fusionner monologiquement au sien. Car elle les reproduit tels quels: me voici, moi $R R$, dans le rôle de l'éditeur (TdeM 201). En déclarant cela, elle se "plurilinguise» tout en restant fidèle aux différences stylistiques de langage tenu par chacun des sujets narratifs dans leurs textes respectifs. $\mathrm{RR}$ termine le roman textuellement, mais elle ne le parachève pas diégétiquement puisque son travail d'éditrice renvoie le lecteur au roman lui-même.

Processus de REdoublements et non pas de DÉdoublements. Il est ainsi question d'itération plutôt que de répétition. Presque synonyme de répétition, l'itération décrit le retour d'un élément ou d'un semblable dans des contextes différents. Il s'agit d'un phénomène qu'il faut surtout ne pas confondre avec celui du "retour du refoulé» même si celui-ci est similaire dans sa fonction en psychanalyse. Si la répétition est un retour avec une même finalité et une même efficace (sinon le fait même du renforcement), clarifie Marc Angenot, l'itération a probablement une efficace différente ${ }^{13}$. Par efficace, j'entends l'effet immanent d'un énoncé ou d'un texte, effet «idéal" qu'il faut distinguer de l'effet référentiel (par exemple à l'égard du texte réaliste, le rapport de la représentation du monde empirique). Ni effet de réel ni effet de sens, on doit rapprocher l'efficace de la notion aristotélicienne de telos, commele précise Angenot. Or le contexte et l'efficace de chacune des instances narratives dans Trou de mémoire ont des caractéristiques particulières, irréductibles à l'une ou à l'ensemble de celles des autres instances. Ce qui les différencie, par contre, ne devient visible justement que par la mise en relation des contradictions immanentes qui en découlent, dégageant ainsi leur apparente autonomie narrative. Le mode esthétique dominant qui met en relief ces contradictions et ces effets de chiaro-oscuro, dans le roman est produit par l'anamorphose. Bien plus qu'une simple composante diégétique, l'anamorphosisation est un procédé qui fonctionne comme structure de médiation esthétique à la mise en texte et qui, en outre, confère un sens concret à la lecture du roman, rapprochant celle-ci de son écriture. Soulignons toutefois qu'écriture et lecture (même celle-ci sous forme de [ ré] écriture) ne peuvent être confondues l'une 
à l'autre. Il n'est jamais question de moments simultanés d'une même production mais bien de deux pratiques différentes dont les contextes et les finalités distincts les rendent, par contre, inter-dépendantes l'une de l'autre. Premièrement, l'anamorphose rend compte ici de la présence de réalités (ou "vérités») narratives, mais qui sont falsifiées dans leur unicité puisque ce procédé entend également les accentuer dans toute leur ampleur factice en les réitérant plusieurs fois, chaque fois différemment, d'où la démultiplication polyphonique. Ceci relève, en théorie littéraire, d'une problématique de la productivité textuelle à l'intérieur de l'œuvre elle-même: on peut dire ainsi que Trou de mémoire est méta-[inter]textuel. Puis, deuxièmement, l'anamorphosisation oblige le lecteur, piqué au vif par l'aspect déroutant du procédé - par la forme mystérieuse que revêt l'ensemble de cette réalité narrative - à démystifier la condition du scriptible pour pouvoir en prendre pleinement conscience et surtout en rendre raison. Et ceci relève d'une autre problématique, celle de la production du texte.

C'est peut-être dans les termes suivants que se résume au mieux l'esthétique de Trou de mémoire: un art qui rend invisible les images apparentes en leur infligeant une dilatation démesurée, en leur conférant une allure proprement hallucinatoire avant de leur injecter toute signification, déclare R R au sujet du trompe-l'œil en théâtre (TdeM 129). C'est-à-dire, par un double dispositif où le seul déchiffrement possible est en fonction de ce que l'anamorphose masque par sa distorsion. Il devient alors nécessaire de ne plus s'en tenir uniquement aux conditions de la productivité textuelle de son esthétique mais de restituer à celle-ci ses conditions sociales de production: conditions en fonction desquelles il y a processus de distorsion. Derrière l'anamorphosisation se trouve également son catalyseur:

Un romancier a beau imaginer les événements et les personnages les plus invraisemblables (s'exclame RR) il manipule inévitablement des parcelles de réalité. L'imagination littéraire, on le sait, n'est jamais pure de toute coïncidence avec la réalité. Je le sais mieux que quiconque; et je n'ai pas besoin de posséder par caur la clé de toutes les constructions romanesques pour savoir que nul écrivain n'invente à partir de rien. Qu'il l'avoue ou non, il ne peut pas faire autrement qu'utiliser des points de départ dans la réalité.

(TdeM 123-124)

Trou de mémoire est une œuvre où l'Histoire, même si elle est constamment présente, ne s'inscrit que de biais, obliquement, délibérément transformée, et en un mot: anamorphosée. Les références directes à l'époque contemporaine n'abondent pas dans le texte, sauf au début du roman, et celles à l'Histoire en général sont sous le signe d'une conception anarchisante, comme en témoignent les nombreux renvois à des théoriciens de l'anarchie. On ne peut s'empêcher de penser, dans l'optique bakhtinienne de la carnavalisation, aux transformations que subissent les manifestations du Carnaval 
au XVI' siècle avant que celui-ci ne perde complèiement son caractère sociohistorique d'institution populaire. C'est l'époque marquée par le début des censures, puis des interdictions de ses manifestations collectives, surtout lorsque ces dernières servent de plus en plus de prétexte à l'émeute urbaine, voire à la révolte ouverte et inconditionnelle. Je songe, entre autres, au Carnaval de Romans en France, rendu célèbre dans l'ouvrage du même nom par l'historien Emmanuel Le Roy Ladurie (publié chez Gallimard en 1979), où, en février 1580 , le joyeux théâtre populaire et spontané tourne à l'émeute, à la fête sanglante et à la tuerie. Sur le plan socio-culturel, le Carnaval de la place publique et universelle cède alors le terrain à une manifestation d'apparat et de palais, replié sur la privatisation et la domesticité. $\mathrm{ll}$ y a répression de tous les aspects traditionnels de la culture populaire que des sanctions officielles de plus en plus centralisatrices et absolutistes récupèrent et régimentent graduellement en une culture de fêtes de cour, de bals masqués et de parades militarisées (plus tard, on assiste, à la folklorisation proprement dite du Carnaval). J'ajouterai que ce n'est que dans cette optique, impossible à développer ici, que peut être abordé le problème du «baroque» en général, à plus forte raison pour ce qui est de Trou de mémoire, si l'on ne veut pas déraper dans des applications mécanistiques en matière de théorie esthétique ${ }^{14}$.

Quant à la conjoncture du Québec dans les années soixante — période qui conditionne Trou de mémoire, lequel, rappelons-le, est publié en 1968 -, il n'est pas dans mes propos d'en reprendre ici le détail. Réitérons simplement que c'est à partir de 1960 que s'organise de manière systématique le mouvement séparatiste québécois qui vise l'indépendance nationale. C'est l'époque des diverses manifestations populaires et estudiantines, et celle du FLQ, qui culmine avec les événements d'octobre 1970 et l'application de la loi sur les mesures de guerre de la part du gouvernement fédéral pendant plusieurs semaines; non seulement au Québec mais dans tout le Canada. Le début de la décennie témoigne d'une vaste activité réformatrice au sein des diverses institutions québécoises: étatisation (du moins partielle) de l'enseignement, où l'Église perd une grande partie de son contrôle sur le système d'éducation; formation de nouvelles sociétés et nationalisation d'entreprises privées; reprise en main par l'État de la sécurité sociale (jusqu'alors liée à la traditionnelle "charité chrétienne»); rapprochement France-Québec dans le domaine culturel; etc. La Révolution tranquille est cependant de courte durée, courtcircuité par l'inflation croissante, le retour majoritaire de l'Union Nationale aux législatives de 1966, ainsi que la recrudescence de l'influence des ÉtatsUnis dans tous les secteurs de la société, plus particulièrement aux niveaux économiques et financiers. Les dernières années de la décennie sont marquées par une stagnation progressive de l'économie et par le ralentissement de l'effervescence du processus réformateur.

Or, pour retrouver des rapports entre la structure romanesque de Trou de mémoire et les structures de la société québécoise de l'époque, on est obligé de réorganiser le roman "anachronologiquement", c'est-à-dire de le lire «en arrière", de la fin au début, par rapport à la progression de l'histoire du Québec de 1960 à 1970. Il y a renversement des rapports: le roman se 
présente littéralement à l'envers, la tête en bas, par rapport à sa référence historique immédiate. C'est ce que j'ai cherché à schématiser par le synopsis en annexe. Mais c'est l'inversion des correspondances, non les correspondances elles-mêmes, qui constituent les structures médiatrices de Trou de mémoire. Et la nature de ces structures de médiation n'est pas textuelle, mais discursive ${ }^{15}$. Le renversement renvoie aux conditions de formations sociales dans lesquelles l'œuvre est produite, et non aux déterminations immédiates (les correspondances de faits) qui, elles, renvoient à une problématique de la productivité textuelle. Dans Trou de mémoire, cette productivité textuelle est esthétiquement anamorphotique, au sens propre du terme, puisqu'il n'y $\dot{a}$ correspondance effective, en bonnes proportions, qu'après avoir effectué une lecture simultanément anoptique des structures du roman et catoptique des structures de société, sauf dans le cas des notes de l'éditeur et du récit de RR comprenant déjà la discussion sur le tableau des Ambassadeurs ${ }^{16}$.

C'est en cette qualité de renversement qu'il y a ici perception carnavalisante. On y retrouve en tous cas l'un des cinq caractères fondamentaux du Carnaval relevés par Bakhtine et dont André Belleau en a fait l'admirable synthèse ${ }^{17}$. Il s'agit en particulier d'un trait qui se rapproche du phénomène de "rabaissement» à l'étage corporel inférieur. Ceci se manifeste par une problématique propre à l'œuvre en général de Hubert Aquin, et que j'appellerai historio-sexographique, où le discours de l'Histoire est transposé dans le texte romanesque sous forme de discours de la sexualité. Il serait évidemment nécessaire de consacrer un article à lui seul pour bien cerner le sujet en termes critiques. En revanche, il convient de souligner, et avec force, que dans ses rapports avec l'Histoire, la qualité terroriste conférée au thème de la sexualité dans Trou de mémoire. est un prisme (de violence) anamorphotique qu'il ne faut surtout pas confondre avec une vision carnavalesque proprement dite. Terrorisme et sexualité, traversant les structures sociales et les structures textuelles, ont pour fonction d'être une constante thématique de la violence à divers degrés ${ }^{18}$. Ainsi sont figurés, dans le roman, l'échec national québécois sous forme d'échec sexuel, ou encore, le terrorisme socio-politique sous forme de violence sexuelle. Notons également que tous les thèmes de la violence sexuelle, qu'ils apparaissent en tant qu'échec ou viol, et toutes les manifestations consistant à défier la norme institutionnelle de l'hétérosexualité, comme l'homosexualité (RR), la transexualité (RR feignant être Magnant), ainsi que diverses formes d'expressions (comme la masturbation - rappelons que trois des cinq rapports sexuels entre Magnant et Joan sont des masturbations, $\mathrm{y}$ inclus la scène du meurtre -), vont de pair avec le thème de la condition du peuple dominé et colonisé telle que l'auteur de Trou de mémoire la conçoit.

Mon comportement sexuel est à l'image d'un comportement national frappé d'impuissance, déclare Magnant: plus ça va, plus je sens bien que je veux violer... Faire l'amour normalement ne m'intéresse plus vraiment. Je ne sais plus ce qui se produit. Ce désenchantement ressemble trop à une phobie d'impuissance. Fatigué, je rêve à la plénitude du viol - comme les mystiques doivent aspirer à l'extase divine ou à l'apparition... 
Pour se guérir de son impuissance, il est obligé de devenir terroriste car l'acte même de semer la terreur ressemble impudiquement à tout ensemencement du ventre, à cette différence toutefois qu'il ne tient nullement compte de la mutualité du plaisir; c'est un viol! (TdeM 116-17). Ainsi, Magnant justifie d'une part, des activités politiques et un choix de comportement sexuel violents par lesquels il cherche le triomphe destructeur (TdeM 117), ce qui le pousse finalement à assassiner Joan. D'autre part, il annonce le viol de Rachel, en déclarant que sa vraie puissance (le viol) se manifeste dans la nuit blanche des obsédés, dans la rue où il commence à rôder quand les autres couvrent leur magie noire [ allusion à Ghezzo-Quénum ] sous la clandestinité des draps (TdeM 117). Ainsi, sous les signes du "terrorisme sexuel", le meurtre de Joan en arrive à représenter une tentative ratée de conquête nationale, qui est reliée dans le texte du roman à la défaite des événements historiques de 1837-38. Mais en assassinant Joan, Magnant provoque sa propre défaite, en engendrant comme les Patriotes l'histoire d'un peuple sevré de combats et presque mort de peur, à force d'éviter la violence (TdeM 87). En revanche, ce qui représenterait une nouvelle tentative de reconquête, relié au contexte contemporain dans Trou de mémoire - et à quel prix! -, c'est le viol de Rachel. J'ai changé mon nom, écrit-elle, clôturant le roman, je porte un enfant qui s'appellera Magnant - et jusqu'au bout, je l'espère, et sans avoir peur de son nom. Et je veux que mon enfant soit plus heureux que son père et qu'il n'apprenne jamais comment il a été conçu, ni mon ancien nom... (TdeM 204). Dans cette même visée, l'homosexualité de RR ne peut alors inscrire qu'une variation du thème du viol (celui de la violation des mœurs officielles), conçu comme une sorte de "sexualité terroriste»"

Bref, Trou de mémoire est une vision du monde qui, dans l'ensemble, est particulièrement torturée, voire tragique à bien des égards et qui, sous cet angle, n'a plus rien à voir avec la pensée bakhtinienne d'une littérature carnavalisée, fondée sur la relativisation joyeuse ou parodique des langages ou d'autre chose ${ }^{20}$. En sont conservés, par contre, les aspects carnavalisants en tant que technique de mise en structure romanesque. Cet état de choses relève des conséquences socio-culturelles qui relient, dans l'histoire du roman, les modalités polyphoniques de mises en instance narrative à la carnavalisation littéraire. Il n'est pas non plus uniquement du pur hasard que l'anamorphose signifie une lecture anoptique - du bas vers le haut -, en opposition fondamentale à la vision carnavalesque, lecture catoptique qui cherche à englober à la fois le «bas» et le " haut». La superimposition des deux procédés dans Trou de mémoire opère une eśpèce d'anamorphosisation carnavalisante si l'on peut dire ainsi, et dont il convient de resituer l'historicité en termes du problème de circulation culturelle.

Connexe à ce problème, remarquons ce qu'écrit André Belleau:

Si la carnavalisation désigne la transposition dans la littérature de la culture populaire conçue comme vision complète du monde et non simplement la survivance textuelle de résidus carnavalesques, c'est obliger de s'interroger de façon critique sur la pertinence actuelle du concept de culture populaire tel que défini naguère par 
Bakhtine dans les pays où le centralisme bourgeois autoritaire a terminé son ceuvre de destruction et de folklorisation de la culture populaire $^{21}$.

Il faudrait ainsi questionner les cultures hyperindustrialisées et le rôle du roman contemporain aux côtés des modes de production de masse. Car à moins d'effectuer un syncrétisme parfaitement anaxiologique, il sera nécessaire de distinguer, l'une par rapport à l'autre, "culture élitiste", "culture populaire", "culture de masse» (bourgeoise) et peut-être aussi «culture industrielle» à tendance plus particulièrement populiste et qui ne doit pas être confondue à "culture ouvrière". Pour ce qui est justement de la littérature et du roman, la théorisation à ce sujet reste encore aujourd hui à être réalisée de manière systématique, et pour laquelle une étude conjoncturée et critique des travaux de Bakhtine, Lukács, Benjamin et Adorno ne serait qu'un premier point de départ ${ }^{22}$.

En revanche - et ceci dans l'esprit d'interaction entre les divers domaines dans l'unité de la production culturelle - on retrouve dans les travaux sociologiques de Pierre Bourdieu une notion qui est particulièrement pertinente en regard du problème de la dynamique interactive entre pratiques culturelles diverses: l'habitus. J'en reprends ici les caractéristiques essentielles telles qu'elles sont résumées par Bourdieu lui-même:

Histoire incorporée, faite nature, et par là oubliée en tant que telle, l'habitus est la présence agissante de tout le passé dont il est le produit: partant, il est ce qui confère aux pratiques leur indépendance relative par rapport aux déterminations extérieures du présent immédiat...

...l'habitus comme sens pratique opère la réactivation du sens objectivé dans les institutions: produit du travail d'inculcation et d'appropriation qui est nécessaire pour que ces produits de l'histoire collective que sont les structures objectives parviennent à se reproduire sous la forme des dispositions durables et ajustées qui sont la condition de leur fonctionnement, l'habitus... est ce qui permet d'habiter les institutions, de se les approprier pratiquement, et par là de les maintenir en activité, en vie, en vigueur, de les arracher continûment à l'état de lettre morte, de langue morte, de faire vivre le sens qui s'y trouve déposé, mais en leur imposant les révisions et les transformations qui sont la contrepartie et la condition de la réactivation ${ }^{23}$.

Je ne ferai qu'esquisser en quelques lignes les conséquences que suscite, à l'égard de la critique littéraire, la mise en relation de la notion d'habitus avec le problème du rapport entre la carnavalisation et la polyphonie littéraire dans l'histoire du roman. Les diverses manifestations carnavalesques de la post-Renaissance, c'est-à-dire en leur composantes littéraires et telles que Bakhtine prétend les retrouver dans le roman à partir du XVII' siècle époque à laquelle apparaissent également des éléments polyphoniques dans la littérature - constitueraient non seulement des inscriptions de restes de 
«culture populaire» mais surtout une pratique culturelle dont la caractéristique se définirait comme une sorte d'habitus déclassé, et au sujet duquel Bourdieu se réfère en termes de l'effet don Quichotte. Soit, un processus de déclassement en vertu de la conversion diachronique (historique) et synchronique (transhistorique) d'une pratique qui ressortit à un mode particulier de production culturelle, économiquement déterminée, celle du "populaire» et de l'officieux - pour reprendre la rhétorique bakhtinienne - , en une pratique différente relevant d'un autre mode de production, bourgeoisement autoritaire et dominante, qui la réactive, et à laquelle se grefferait plus tard, peut-être bien, un caractère populiste. Une pratique, enfin, qui trouverait ultérieurement ses légitimations textuelles et narratologiques dans et par la polyphonie, telle que celle-ci est définie par Bakhtine au sujet du roman dostoïevskien.

Dans ces conditions, la pratique esthétique de l'anamorphosisation dans Trou de mémoire fonctionnerait à titre de représentation artistique de ce phénomène social de conversion. Et cela, sans omettre de rappeler que l'anamorphose en peinture n'est pas un art "populaire" mais un art de la "haute" culture, et dont l'historicité précède ce que l'idéologie centralisatrice baroque vient récupérer des concepts artistiques du style maniériste, au XVII ${ }^{\circ}$ siècle. Il reste alors à se demander, dans une telle optique, dans quelle mesure on peut encore parler de "texte carnavalisé" ou de "roman carnavalesque" dans notre type de société ${ }^{4}$. Car si le dialogique de la vision carnavalesque et ses conséquences littéraires, la carnavalisation, témoigne d'une dialectisation à l'intérieur du phénomène de circulation culturelle au Moyen Âge et sous la Renaissance (ceci, si l'on veut être fidèle aux préceptes de Bakhtine), il n'en est pas de même avec la polyphonisation littéraire. Le dialogique polyphonique n'est pas dialectique, et à plus forte raison ne l'est pas celui des manifestations polyphoniques contemporaines dans le roman des dernières vingt ou vingt-cinq dernières années, c'est-à-dire l'après-Nouveau Roman. Il s'agit là d'une performance narratologique dont la multiplicité polyphonique de plans et de voix est essentiellement méta-référentielle, tel qu'en témoigne l'exemple de Trou de mémoire. Le processus transformationnel de la polyphonie dont il est question chez Bakhtine, se replie sur lui-même, devient méta-textuel, parfois méta-fictionnel.

A l'instar d'une cuvre telle que Trou de mémoire, la polyphonie contemporaine recèle ainsi tous les indices de la résurgence d'un esthétisme à outrance; s'y greffe un dialogisme renfermé sur son propre mouvement, court-circuitant le courant de la transformation dialectique, et où les processus narratifs se bloquent dans une espèce de surdétermination dialogisante ${ }^{25}$. C'est un processus qui détourne le dialectique et, à la limite, l'étouffe. Il s'en dégage une deshistoricisation par laquelle semble se mettre en place un état d'immutabilité historique, et qui consiste à dire que seules peuvent changer les formes en lesquelles le processus historique s'articule et se manifeste. L'apport téléologique de l'Histoire, son développement, le devenir des choses bakhtinien, sont par contre évacués. De sorte que la polyphonie semble alors être la source mystérieuse et inépuisable de son propre dynamisme, créant elle-même la transformation de ses propres formes. 
Ceci renvoie d'emblée au problème du redoublement dans sa signification d'accroissement, d'accumulation. Le redoublement est un phénomène qui sera toujours porté vers une suresthétisation de ce type. Trou de mémoire n'est qu'une des illustrations de la production romanesque contemporaine et qui annonce quelques exemples extrêmes, tels que Se una notte d'inverno un viaggiatore d'Italo Calvino, Die unendliche Geschichte de Michael Ende, Ararat de Don M. Thomas... Tout ceci ressortit à une problématique de la fétichisation dont l'anamorphose est l'un des produits esthétiques. C'est en ces termes que l'on retrouve une contradiction dominante dans la pratique d'anamorphosisation chez Hubert Aquin. Afin d'élucider cette contradiction, les propos suivants de Gilles Deleuze et Félix Guattari méritent d'être cités:

le capitalisme ne cesse de fuir par tous les bouts. Ses productions, son art, sa science forment des flux décodés et déterritorialisés qui ne se soumettent pas seulement à l'axiomatique correspondante, mais qui font passer certains de leurs courants à travers les mailles de l'axiomatique, par-dessous les recodages et les reterritorialisations. A leur tour, des groupes-sujets dérivent par rupture des groupes assujettis... Toujours prêt à agrandir ses limites intérieures, le capitalisme reste menacé par une limite extérieure qui risque d'autant plus de lui venir et de le fendre du dedans que les limites intérieures s'agrandissent ${ }^{26}$.

Et, en termes plus précisément «schizo-analytiques», ces conditions font que

l'actualité d'une potentialité révolutionnaire s'explique moins par l'état de causalité préconscient dans lequel elle est pourtant comprise, que par l'effectivité d'une coupure libidinale à un moment précis, schize dont la seule cause est le désir, c'est-à-dire la rupture de causalité qui force à réécrire l'histoire à même le réel et produit ce moment étrangement polyvoque où tout est possible ${ }^{27}$.

"Trompe-l'œil» (dans Trou de mémoire), la polyphonie contemporaine se situe également au niveau d'un "trompe-l'esprit» que je n'hésiterai pas à qualifier à la limite d'idéologie frauduleuse et dont l'illusion consiste, à un moment précis, à rendre tout étrangement possible - même si cette possibilité est parfaitement inexistante. Et si l'on cherchait à cerner une théorie de l'économie esthétique dans une telle conjoncture, il faudrait se reporter à la théorie de la plus-value dans le système économique du capital porteur d'intérêt. $A$ partir de la formule $A-A^{1}$, réduction extrême de $A-M-A^{1}$ où l'argent produit de l'argent sans passer par le procès de la transformation matérielle du capital, et par lequel $\mathrm{A}^{1}=\mathrm{A}+\Delta \mathrm{A}$ (c'est-à-dire, l'argent créant l'argent), on pourrait reconstituer une formule, peut-être trop simple mais en tous cas symptomatique, du suresthétisme contemporain. Dans cette reformulation, on obtiendrait $\mathrm{d}-\mathrm{Di}-\mathrm{D}^{1}$ où $« \mathrm{~d} »$ représente le dialogue, "Di» le dialectique et " $\mathrm{D}^{1}$ " le dialogisme polyphonique, et où « Di» disparaît pour faire place à $D^{1}=d+\Delta d$ : le dialogisme produisant encore des dialogues sans passer par le procès de transformation dialectique. 
En guise de conclusion, je voudrais revenir au sujet du problème de la carnavalisation dans les sociétés industrielles dites «avancées», en citant un passage de l'article d'André Belleau: on pourrait concevoir la carnavalisation à la façon d'Auerbach dans Mimesis: l'irrupton du bas, à l'origine populaire, comique, dans le haut, sérieux, problématique, voire tragique ${ }^{28}$. À mon sens, la visée idéologique d'Auerbach ne peut pas être comparée à celle de Bakhtine. Pour ce dernier, le genre romanesque fait reposer ses bases constitutivement 'culturelles sur une interdiscursivité qui cherche à rendre compte de l'ensemble des divers types de discours sociaux, en leur devenir et ce que cela signifie en termes du développement de l'Histoire. À l'égard de la disparition de la littérature "populaire" aujourd'hui (au sens bakhtinien du terme "populaire" ), refoulée et folklorisée par la bourgeoisie puis détournée et étouffée par la littérature industrielle de masse, comme dit Belleau ${ }^{29}$, il serait peut-être utile d'introduire une nuance qui permettrait de préciser la « vision carnavalesque" par rapport à une "perception carnavalisante". La vision carnavalesque n'évacue jamais le discours auquel elle se réfère et qu'elle carnavalise, au cas contraire il n'y aurait pas de phénomène de rapprochement entre les oppositions qu'engendre l'interaction dialogique, mais justement simple effet de substitution. La perception carnavalisante non plus, mais si elle conserve l'ambivalence, voire l'ambiguiité qui en découle, par contre elle n'en garde que l'habitus de ses éléments carnavalesques, décollés de leur signification sociale ou, plus exactement, comme j'ai cherché à l'expliquer plus haut, dont la socialité a été sujette au phénomène de conversion. En revanche, l'idée auerbachienne de l'irruption du bas dans le haut est, à mon avis, essentiellement monologique et donc foncièrement incompatible avec l'esprit bakhtinien. Le principe moniste, pour Bakhtine et que je reprends ici en regard du concept du réalisme chez Auerbach, est la transformation de l'unité de la substance en un principe (hégélien) d'unité de la conscience (romanesque occidentale, il me semble pour Auerbach). La sphère d'existence dans laquelle se développe la nature dialogique de la pensée humaine et de l'idée, précise bien Bakhtine, n'est pas l'unité de la conscience individuelle bourgeoise, mais celle des rapports dialogiques avec les idées d'autrui, dans l'unité de la substance, matérielle et historique.

Le polyphonique contemporain n'en est pas pour autant du monologisme déguisé; il garde, accentue même le caractère fondamental d'interaction dialogique et en multiplie les formes narratives et les modalités esthétiques sous lesquelles ce caractère se manifeste dans un texte romanesque, comme Trou de mémoire notamment. Le problème est qu'il y a court-circuitage de l'apport téléologique matérialiste, de Bakhtine, du développement historique, c'est-àdire de sa lecture englobante de l'Histoire. 


\section{ANNEXE}

Correspondances entre structures textuelles et structures de société

TEXTE

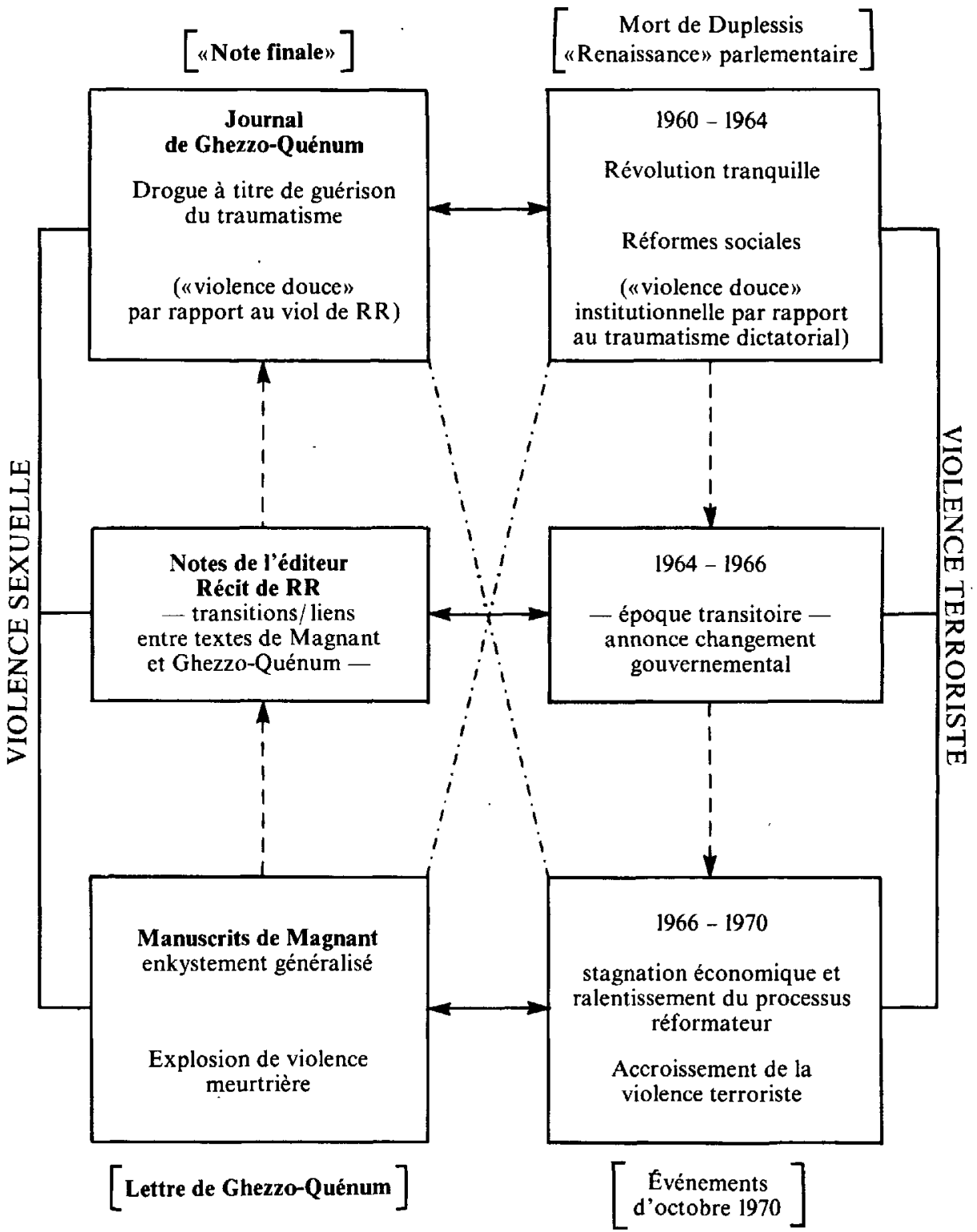

$\longrightarrow$ : Structuration de la représentation factuelle

$-z_{-} \rightarrow$ : Temporalisation chronologique de la diégèse fictionnelle et de l'histoire -..-... : Correspondance événementielle 
1. Voir pour une première approche à la problématique soutenue ultérieurement dans ma thèse de doctorat, "Tres tristes tigres or the Treacherous Play on Carnival ", Ideologies \& Literature. A Journal of Hispanic and Luzo-Brazilian Literatures, Vol. 3, no 15 (janvier-mars 1981), p. 33-56. Voir également mes articles, «Mikhail Bakhtin and Contemporary Narrative Theory" Revue de l'Université d'Ottawa/University of Ottawa Quarterly, Vol. 53, $\mathrm{n}^{\circ} 1$ (janvier-mars 1983), p. 51-65; "Critique de la (dé)raison polyphoniquen, Etudes françaises, Vol. 20, $\mathrm{n}^{\circ} \mathrm{I}$ (printemps 1984), p. 45-56; "Polyphonic Theory and Contemporary Literary Practices", Studies in Twentieth Century Literature, Vol. 19, no 1 (automne 1984), p. 75-87; ainsi que «Théorie et littérature comparée: l'instance dialogisée de la pratique littéraire", Neohelicon, à paraître.

2. Tel qu'effectué par l'Édition critique de l'œuvre d'Hubert Aquin, Département d'études littéraires, Université du Québec à Montréal.

3. André Belleau, «Carnavalisation et roman Québécois: mise au point de l'usage d'un concept de Bakhtine», Études françaises, Vol. 19, n 3 (hiver 1983-1984), p. 51.

4. J'utilise l'édition du Cercle du livre de France, Ottawa, 1968. Les citations dans le texte sont signalées par le sigle "TdeM» et le numéro de la page correspondante.

5. Jurgis Baltrusaitis, Anamorphoses ou magie artificielle des effets merveilleux, Paris, Olivier Perrin, 1969, p. 5-6.

6. Se basant sur l'ouvrage de Danicl Barbaro, Pratica della perspettiva (1559), Baltrusaitis explique la mise en pratique de l'anamorphose en peinture, qui est à l'inverse de l'effet qu'elle produit: la première phase consiste à composer l'image telle qu'elle doit être perçue... la seconde procède à la déformation, mécaniquement avec la lumière. Ibid., p. 34 .

7. Ibid., p. 34.

8. Tel que cité par Baltrusaitis, Ibid., p. 34-35.

9. Je ne reviendrai pas sur l'ensemble de la problématique de la colonisation dans l'œuvre d'Aquin; elle a été longuement traitée et discutée par de nombreux chercheurs. Je renvoie toutefois à cet ouvrage capital d'Albert Memmi, Portrait du colonisé suivi de Les Canadiens français sont-ils des colonisés?. Montréal, l'Étincelle, 1972. Voir également du même auteur, I'Homme dominé. Paris. Petite Bibliothèque Payot, 1968, ainsi que le livre de Jean Bouthillette, le Canadien français et son double, Ottawa, l'Hexagone, 1972.

10. Jean-Louis Ferrier, Holbein: Les Ambassadeurs. Anatomie d'un chef-d'œeuvre, Paris, Denoël/Gonthier, 1977, p. 40.

11. John Berger, Ways of Seeing, Londres, B.B.C. et Penguin, p. 90 et s.

12. Voir Arnold Hauser, The Social History of Art, Vol. 2 ( Renaissance, Mannerism, Baroque»), Londres, Routledge \& Kegan Paul, 1962.

13. Marc Angenot, Glossaire pratique de la critique contemporaine, Montréal, Hurtubise, 1979 , p. 114.

14. Voir mon article, «Critique de la (dé)raison polyphonique», op. cit., pour une esquisse sur le problème du "baroque" dans le don Quichotte.

15. Voir l'ouvrage suivant, important pour la sociocritique, d'Edmond Cros, Théorie et pratique sociocritiques, Montpellier, C.E.R.S. / Paris, Editions Sociales, 1983.

16. Cette correspondance rejoint le procédé du Cinémascope par lequel sont utilisés simultanément deux anamorphoseurs, l'un projetant l'image horizontalement, l'autre verticalement, évitant ainsi toute déformation.

17. Belleau, op. cit.

18. Consulter à ce sujet Sex and Violence in the Canadian Novel: The Ancestral Past, de John Moss, Toronto, McLelland and Stewart, 1977, où, du reste, il n'est question que du roman canadien anglophone. Voir également les Actes du Colloque sur la «Violence dans le roman canadien depuis 1960 ", éd. par Virginia Harger-Grinling et Terry Goldie. Memorial University, Terre-Neuve, s.d. 
19. Il faudrait - ainsi - noter, au sujet de la problématique historio-sexographique chez Hubert Aquin, qu'il s'agit bien dans son œuvre d'une thématique récurrente dont le tracé idéologique est fortement teinté d'un apport traditionnellement anti-féministe (tout en l'étant pas ouvertement), et à l'intérieur duquel s'inscrit également quelque chose de misogyne. Une lecture féministe des romans d'Aquin se fait attendre.

20. Belleau. op. cit., p. 64. On notera que Belleau n'inclut pas l'œuvre d'Aquin parmi ses exemples de littérature carnavalisée québécoise.

21. Ibid., p. 53.

22. Remarquons que l'historien Robert Muchembled considère la «Petite bibliothèque bleue de Troyes" comme le premier phénomène de culture (littéraire) de masse, en France en tous cas. Voir son ouvrage Culture populaire et Culture des élites, Paris, Flammarion. 1979.

23. Pierre Bourdieu, le Sens pratique, Paris, Minuit, 1980, p. 94, 96. Consulter également du même auteur, la Distinction. Critique sociale du jugement, Paris, Minuit, 1979.

24. Cette question est valable aussi bien pour la littérature québécoise des vingt-cinq dernières années, que, notamment, pour la littérature latino-américaine dite du "Boom». Par literatura del "Boom» est entendue la véritable explosion littéraire hispanique à partir du début des années soixante. Deux ouvrages intéressants à ce sujet qui méritent d'être cités; José Donoso, Historia personal del «boom», Barcelone, Seix Barral, 1972, et Hernan Vidal, Literatura hispanoamericana e ideologia liberal: Surgimiento y crísis (una problematica sobre la dependencia en torno a la narrativa del boom), Buenos Aires, Hispamérica, 1976. Et, tournant autour du même problème, voir l'article de Jean Franco, «Dependency Theory and Literary History», Minnesota Review, Vol. 5 (1975), p. 65-80.

25. La surdétermination n'a rien à voir avec la dialectisation; c'est un phénomène de société divisée en classes dans laquelle, comme l'indique Bourdieu, tous les produits d'un agent déterminé parlent inséparablement et simultanément, par une surdétermination essentielle, de sa classe (ou plus précisément, de sa position dans la structure sociale et de sa trajectoire, ascendante ou descendante) et de son corps, ou, plus précisément, de toutes les propriétés, toujours socialement qualifiées, dont celui-ci est porteur. Voir le Sens pratique, op. cit., p. 133.

26. Gilles Deleuze/ Félixe Guattari, Capitalisme et Schizophrénie. L'Anti-Oedipe, nouv. éd. augm. Paris, Minuit, 1975. p. 451.

27. Ibid., p. 453-54.

28. Belleau, op. cit., p. 62.

29. Ibid., 